\title{
Condição de saúde bucal de dependentes químicos em processo de reabilitação: um estudo transversal
}

\author{
Oral health condition of drug addicts in the process of rehabilitation: a cross-sectional study
}

Estado de salud bucal de los drogodependientes en proceso de rehabilitación: un estudio

transversal

Recebido: 15/01/2021 | Revisado: 16/01/2021 | Aceito: 19/01/2021 | Publicado: 24/01/2021

\author{
Andrea Sayuri Silveira Dias Terada \\ ORCID: https://orcid.org/ 0000-0002-9557-6664 \\ Universidade de Rio Verde, Brasil \\ E-mail: andrea.terada@unirv.edu.br \\ Aline de Oliveira Marques \\ ORCID: https://orcid.org/ 0000-0001-9898-8149 \\ Universidade de Rio Verde, Brasil \\ E-mail: alline.odonto@gmail.com \\ Millena Barroso Oliveira \\ ORCID: https://orcid.org/ 0000-0001-5878-781X \\ Universidade Federal de Uberlândia, Brasil \\ E-mail: millenaboliveira@outlook.com \\ Susana Martins de Souza \\ ORCID: https://orcid.org/ 0000-0002-3189-3871 \\ Universidade de Rio Verde, Brasil \\ E-mail: susanamartins46@gmail.com \\ Marcelo Bighetti Toniollo \\ ORCID: https://orcid.org/ 0000-0003-2167-4568 \\ Universidade de Rio Verde, Brasil \\ E-mail: marcelo.toniollo@unirv.edu.br \\ Luiz Renato Paranhos \\ ORCID: https://orcid.org/ 0000-0002-7599-0120 \\ Universidade Federal de Uberlândia, Brasil \\ E-mail: paranhos.lrp@gmail.com
}

\begin{abstract}
Resumo
Objetivo: Avaliar as condições de saúde bucal de dependentes químicos em processo de recuperação do município de Rio Verde - Goiás. Metodologia: Foi realizado um estudo transversal descritivo, de acordo com as recomendações do Strengthening the Reporting of Observational Studies in Epidemiology (STROBE). Foram analisados 41 questionários de saúde pertencentes a 24 homens e 17 mulheres, pacientes de duas comunidades terapêuticas do município de Rio Verde-Goiás, com faixa etária predominante de 31 a 40 anos. Dados da anamnese, exame clínico intrabucal, índice CPO-D, índice periodontal comunitário e índice de placa corada foram analisados. Os resultados foram expressos por meio de estatística descritiva. Resultados: O CPO-D médio da população em estudo foi de 14,72. Pouco mais da metade dos indivíduos, 53,65\% $(\mathrm{n}=22)$, apresentaram sangramento à sondagem. A higiene bucal de $75,61 \%(n=31)$ foi considerada precária e 48,78\% ( $n=20)$ relataram não fazer uso diário do fio dental. Lesões em mucosa oral esteve presente em 39,02\% ( $\mathrm{n}=16)$ dos pacientes avaliados. Conclusão: De acordo com os resultados obtidos, observou-se condição de saúde bucal desfavorável entre os participantes do estudo, demonstrando a importância da elaboração e emprego de estratégias educativo-preventivas à população de dependentes químicos em processo de recuperação.
\end{abstract}

Palavras-chave: Compostos químicos e drogas; Dependentes químicos; Saúde bucal.

\begin{abstract}
Objective: To evaluate the oral health conditions of drug addicts in the process of recovery in the municipality of Rio Verde - Goiás. Methodology: A descriptive cross-sectional study was carried out, in accordance with the recommendations of the Strengthening the Reporting of Observational Studies in Epidemiology (STROBE). 41 health questionnaires belonging to 24 men and 17 women, patients from two therapeutic communities in the municipality of Rio Verde-Goiás, with a predominant age range of 31 to 40 years, were analyzed. Anamnesis data, intraoral clinical examination, DMFT index, community periodontal index and stained plaque index were analyzed. The results were expressed using descriptive statistics. Results: The average DMFT of the study population was 14.72. Just over half of the individuals, $53.65 \%(\mathrm{n}=22)$, had bleeding on probing. Oral hygiene of $75.61 \%(\mathrm{n}=31)$ was considered poor and
\end{abstract}


$48.78 \%(n=20)$ reported not using floss daily. Lesions in the oral mucosa were present in $39.02 \%(n=16)$ of the evaluated patients. Conclusion: According to the results obtained, an unfavorable oral health condition was observed among the study participants, demonstrating the importance of developing and employing educational-preventive strategies for the population of drug addicts in the process of recovery.

Keywords: Chemical compounds and drugs; Drug addicts; Oral health.

\section{Resumen}

Objetivo: Evaluar las condiciones de salud bucal de personas adictas a las drogas en proceso de recuperación en el municipio de Río Verde - Goiás Metodología: Se realizó un estudio descriptivo transversal, de acuerdo con las recomendaciones del Strengthening the Reporting of Observational Studies in Epidemiology (STROBE). Se analizaron 41 cuestionarios de salud pertenecientes a 24 hombres y 17 mujeres, pacientes de dos comunidades terapéuticas del municipio de Río Verde-Goiás, con un rango de edad predominante de 31 a 40 años. Se analizaron datos de anamnesis, exploración clínica intraoral, índice CPOD, índice periodontal comunitario e índice de placa teñida. Los resultados se expresaron mediante estadística descriptiva. Resultados: El CPOD promedio de la población de estudio fue de 14,72. Poco más de la mitad de los individuos, el 53,65\% ( $\mathrm{n}=22)$, presentaba sangrado al sondaje. La higiene bucal del 75,61\% $(\mathrm{n}=31)$ se consideró deficiente y el 48,78\% $(\mathrm{n}=20)$ informó no usar hilo dental a diario. Las lesiones en la mucosa oral estuvieron presentes en el 39,02\% ( $\mathrm{n}=16)$ de los pacientes evaluados. Conclusión: De acuerdo con los resultados obtenidos, se observó una condición de salud bucal desfavorable entre los participantes del estudio, lo que demuestra la importancia de desarrollar y emplear estrategias educativo-preventivas para la población de drogodependientes en proceso de recuperación.

Palabras clave: Compuestos químicos y fármacos; Dependencias químicas; Salud bucal.

\section{Introdução}

O consumo de substâncias lícitas como o tabaco e o álcool, e ilícitas como cocaína, maconha e crack, por exemplo, vem aumentando nos últimos anos (Albini et al., 2015; Capistrano, Ferreira, Silva, Kalinke, \& Maftum, 2013; Colodel et al., 2009; Costa, Godoy, Gomes, Pereira, \& Lins, 2011; Falcão et al., 2015). Tais drogas são capazes de provocar alterações significativas na vida dos indivíduos. Mudanças nos padrões de higiene e saúde bucal são perceptíveis, reflexo da perda de autoestima, falta de motivação, condições de moradia, bem como comorbidades psicológicas, aos quais estão sujeitos os usuários de substâncias químicas. (Brasil. Secretaria Nacional de Políticas sobre Drogas [SENAD], 2011; Colodel et al., 2009). Dessa forma, como consequência da ausência de autocuidados bucais, lesões cariosas, xerostomia, halitose, estomatites, doenças periodontais, entre outras, são frequentemente observadas na cavidade oral de dependentes químicos (Albini et al., 2015; Colodel et al., 2009; Costa et al., 2011; Falcão et al., 2015; Ferreira et al., 2018).

Sendo assim, cabe ao profissional da área odontológica identificar, interferir e orientar quanto aos efeitos causados pelo uso das drogas e substância ilícitas, bem como instruir acerca da realização de uma correta higienização, no que se diz respeito a escovação e ao uso do fio dental. O cirurgião-dentista, pode ainda, efetuar tratamento multidisciplinar, em prol de minimizar os efeitos deixados pelas drogas nestes pacientes (Cima \& Corrêa, 2014).

Alterações na cavidade bucal podem estar associadas diretamente ao tempo de uso de drogas (Albini et al., 2015), bem como à exposição direta dos tecidos orais a drogas fumadas ou ingeridas, aliadas à falta de higiene e negligência de cuidados bucais. O reconhecimento dos fatores de risco inicia-se na anamnese, antes do início de qualquer tratamento podendo estabelecer o melhor caminho e abordagem pertinentes a esta população (Alves, Nai, \& Parizi, 2013; Cima \& Corrêa, 2014; Colodel et al., 2009; Marques et al., 2016; Ribeiro, Oliveira, Zambolin, Lauris, \& Tomita, 2002).

Observa-se que cada substância detém ação sobre os tecidos bucais. A cocaína e o crack provocam vasoconstrição levando a deficiência do suprimento sanguíneo, causando necrose tecidual capaz de ocasionar lesões como úlceras na boca e erosão no esmalte dentário. A maconha estimula a redução do fluxo salivar na cavidade oral, aumentando a incidência de cárie e doença periodontal em dependentes químicos (Colodel et al., 2009; Seemüller et al., 2015).

Além disso, o uso de álcool, crack, tabaco e cocaína produzem danos que impactam na vida das pessoas, ocasionando alterações de humor, perda da vaidade e falta de motivação em função dos efeitos no sistema imunológico (Costa et al., 2011; Ribeiro et al., 2002). Nesses casos, a autopercepção das condições de saúde bucal, podem refletir negativamente no bem-estar 
funcional, social e psicológico de usuários. (Costa et al., 2011; Ribeiro et al., 2002). Diante do exposto, o presente estudo teve por objetivo avaliar as condições de saúde bucal de dependentes químicos em processo de recuperação do município de Rio Verde - Goiás.

\section{Metodologia}

Um estudo observacional transversal descritivo foi realizado. O trabalho foi elaborado de acordo com as recomendações do Strengthening the Reporting of Observational Studies in Epidemiology (STROBE) (Malta, Cardoso, Bastos, Magnanini, \& da Silva, 2010). Para cumprir os requisitos da resolução 466/12, o projeto de pesquisa foi submetido ao Comitê de Ética em Pesquisa da Universidade de Rio Verde (UniRV), sendo aprovado conforme CAAE: 73516717.4.0000.5077. Todos os participantes que consentiram em participar da pesquisa assinaram o Termo de Consentimento Livre e Esclarecido (TCLE) previamente a coleta de dados.

\subsection{Amostra}

A presente pesquisa foi realizada por meio de dados secundários de fichas clínicas odontológicas de dependentes químicos em processo de recuperação. Pacientes de duas comunidades terapêuticas do município de Rio Verde/Goiás, Comunidade Terapêutica "Gênesis" e Comunidade Terapêutica "Meninas dos Olhos de Deus", foram examinados por acadêmicos de Odontologia da Universidade de Rio Verde - UniRV, que cursavam a disciplina Saúde Coletiva, no ano de 2018.

As fichas preenchidas incorretamente ou com ausência dos dados de interesse foram excluídas da pesquisa. Foram incluídas apenas aquelas que continham dados acerca das variáveis em estudo, tais como, cuidados odontológicos, prevalência de doença cárie e doença periodontal, hábitos de higiene oral e condição de saúde bucal

\subsection{Coleta de dados}

A catalogação e coleta dos dados de interesse foram realizadas por pesquisador treinado, a fim de manter padronização de coleta e critérios para tabulação. Foram recuperados dados da anamnese, exame clínico intrabucal, índice CPO-D, índice periodontal comunitário e índice de placa corada.

\subsection{Análise dos dados}

Para a tabulação e análise dos dados foram confeccionados gráficos utilizando o Software Microsoft Office Excell@. Os resultados foram expressos e analisados por meio de estatística descritiva.

\section{Resultados}

$\mathrm{O}$ presente estudo avaliou prontuários odontológicos de 41 indivíduos dependentes químicos em processo de recuperação. O perfil de sexo e CPO-D está expresso na Tabela 1. A faixa etária predominante foi de 31-40 anos.

Tabela 1. Média do índice CPO-D, dependentes químicos em processo de recuperação, Rio Verde - Goiás.

\begin{tabular}{c|c|c|c}
\hline Gênero & $\mathbf{N}^{\circ}$ & \% & Índice CPO-D \\
\hline Masculino & 24 & 59 & 16,62 \\
Feminino & 17 & 41 & 12,82 \\
\hline Masculino + feminino & $\mathbf{4 1}$ & $\mathbf{1 0 0}$ & $\mathbf{1 4 , 7 2}$ \\
\hline
\end{tabular}

Fonte: Terada, Marques, Oliveira, Souza, Toniollo e Paranhos (2021). 
Em relação ao índice periodontal comunitário, 53,65\% (n=22) dos indivíduos avaliados apresentaram sangramento à sondagem. A presença de bolsa periodontal foi verificada em 39,02\% (n=16) dos indivíduos e em 78,04\% (n=32) dos participantes havia presença de cálculo dentário.

Quanto aos resultados do índice de placa corada, a Figura 1 demonstra os resultados de cada um dos participantes. Desses, 75,6\% (n=31) apresentavam acúmulo de placa bacteriana em mais de 20\% do total de sítios avaliados.

Figura 1. Índice de placa corada, dependentes químicos em processo de recuperação, Rio Verde - Goiás.

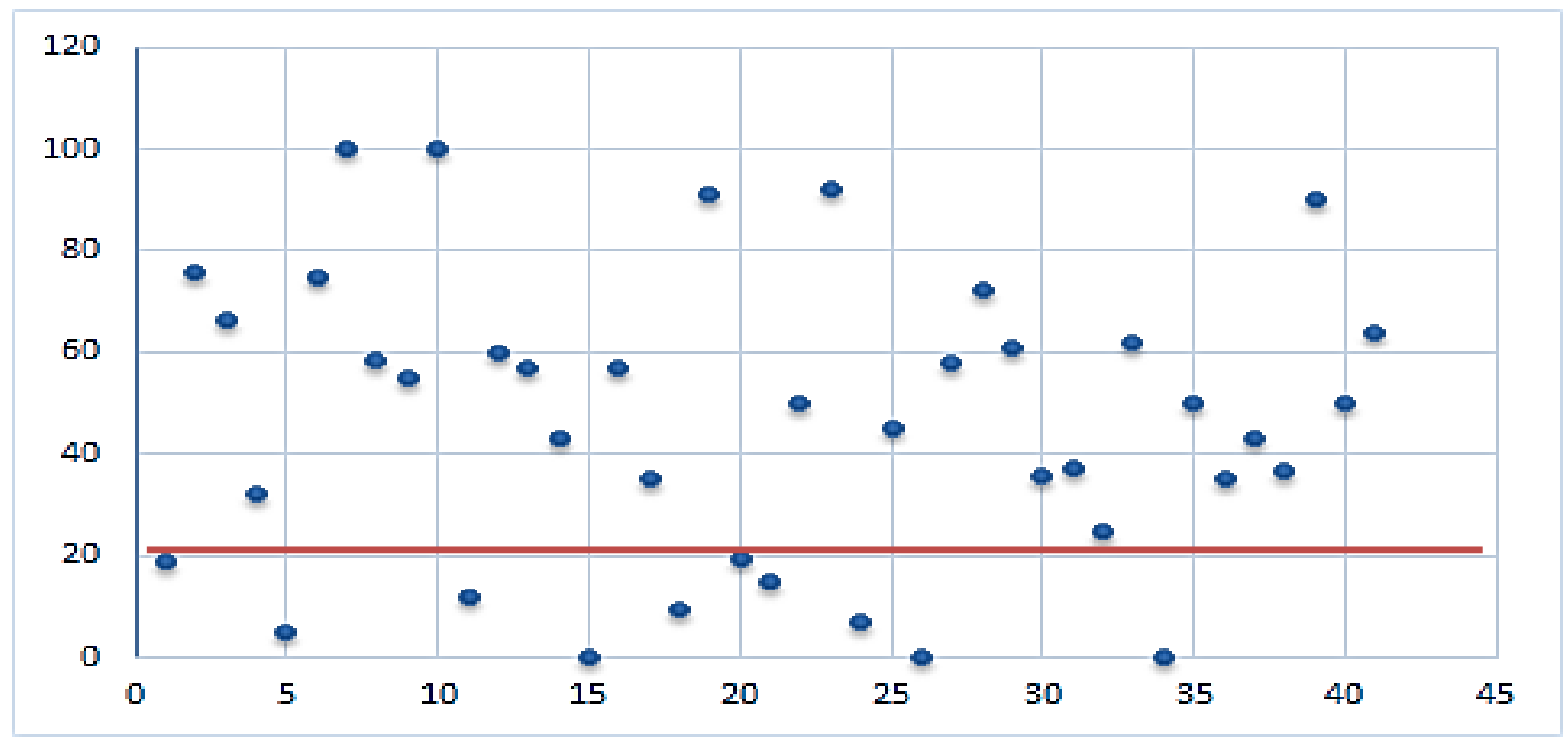

Fonte: Terada et al. (2021).

A condição de saúde bucal dos indivíduos foi considerada como "Boa" em 24,39\% (n=10) dos pacientes, 48,78\% $(n=20)$ e 26,83\% (n=11) apresentaram condição de saúde bucal "Regular" e "Ruim", respectivamente. Aproximadamente metade, $49 \%(n=20)$, relataram não fazer uso do fio dental. Outros $49 \%(n=20)$ faziam uso do fio dental e, 2\% ( $n=1)$ não informaram.

O percentual de escovação dentária diária entre os participantes está expresso na Figura 2. 
Figura 2. Distribuição da porcentagem quanto a frequência de escovação diária, dependentes químicos em processo de recuperação, Rio Verde - Goiás.

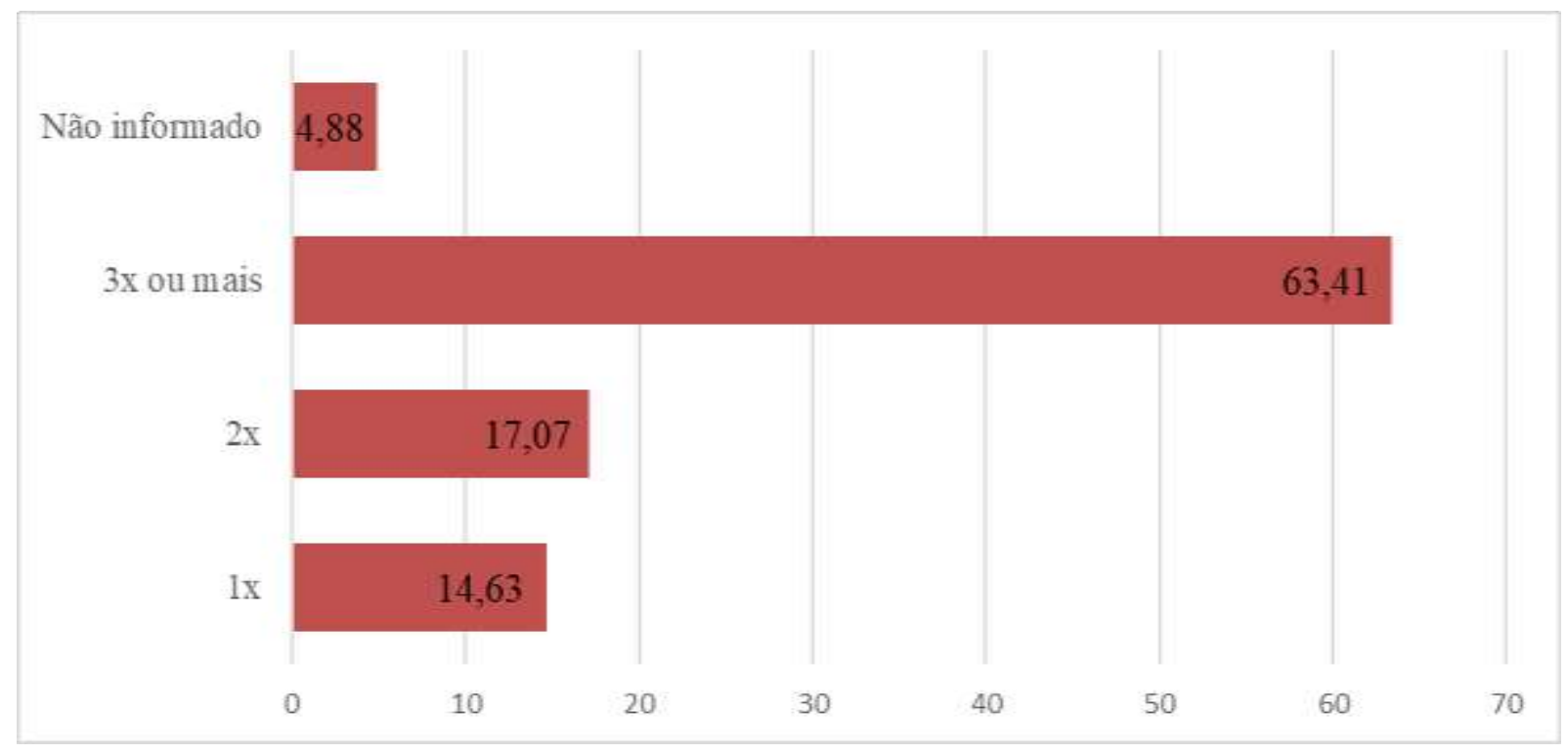

Fonte: Terada et al. (2021).

Em se tratando do registro das condições de tecidos moles dos indivíduos, 39,02\% (n=16) apresentavam alterações na mucosa. Além disso, 48,78\% $(n=20)$ consideravam ter halitose.

Foram extraídas ainda, informações quanto a última visita ao cirurgião-dentista (Figura 3), e perfil de necessidade de tratamento odontológico dos participantes em estudo (Figura 4).

Figura 3. Última visita ao cirurgião-dentista, dependentes químicos em processo de recuperação, Rio Verde - Goiás.

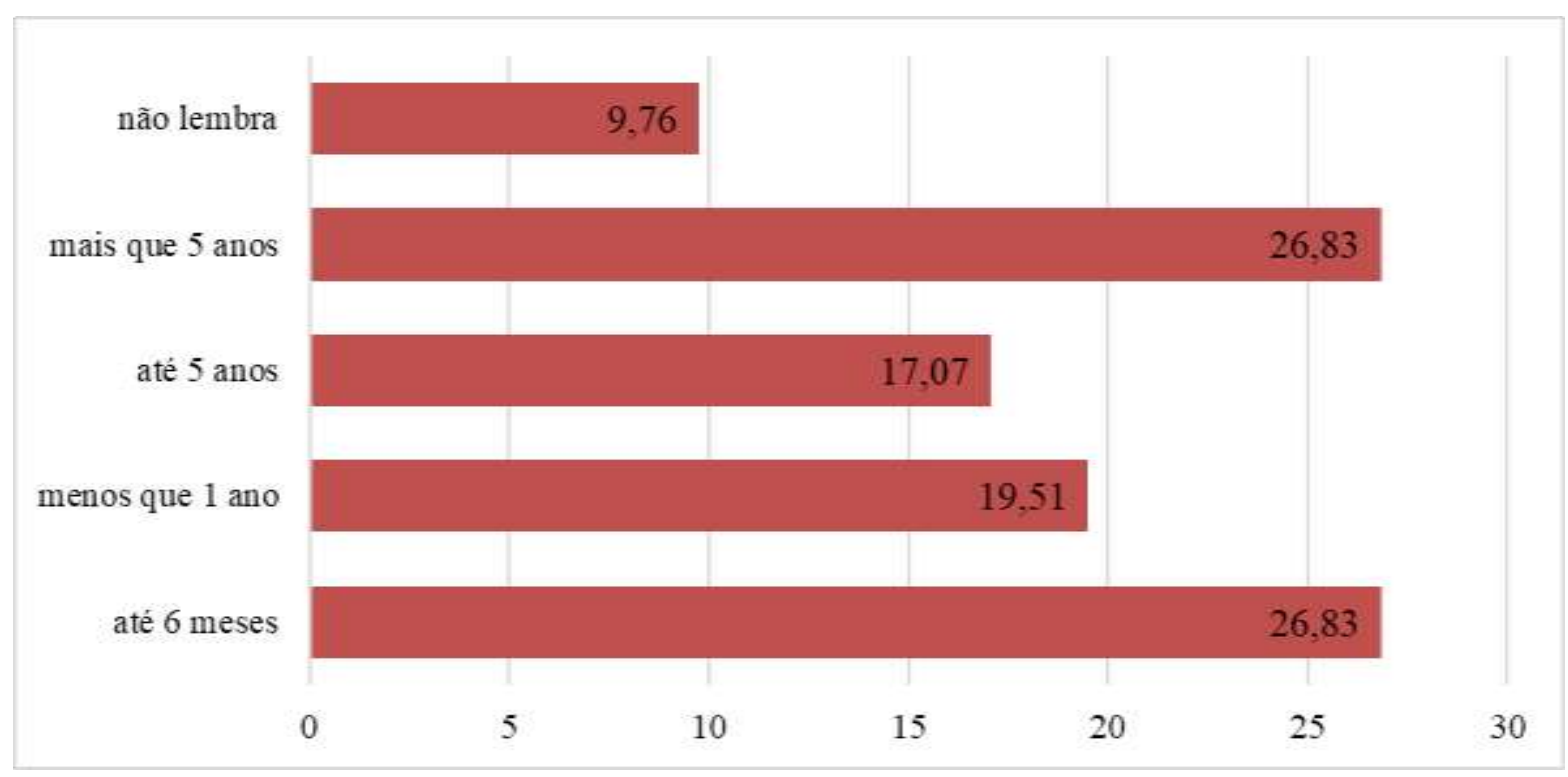

Fonte: Terada et al. (2021). 
Figura 4. Necessidade de tratamento odontológico, dependentes químicos em processo de recuperação, Rio Verde - Goiás.

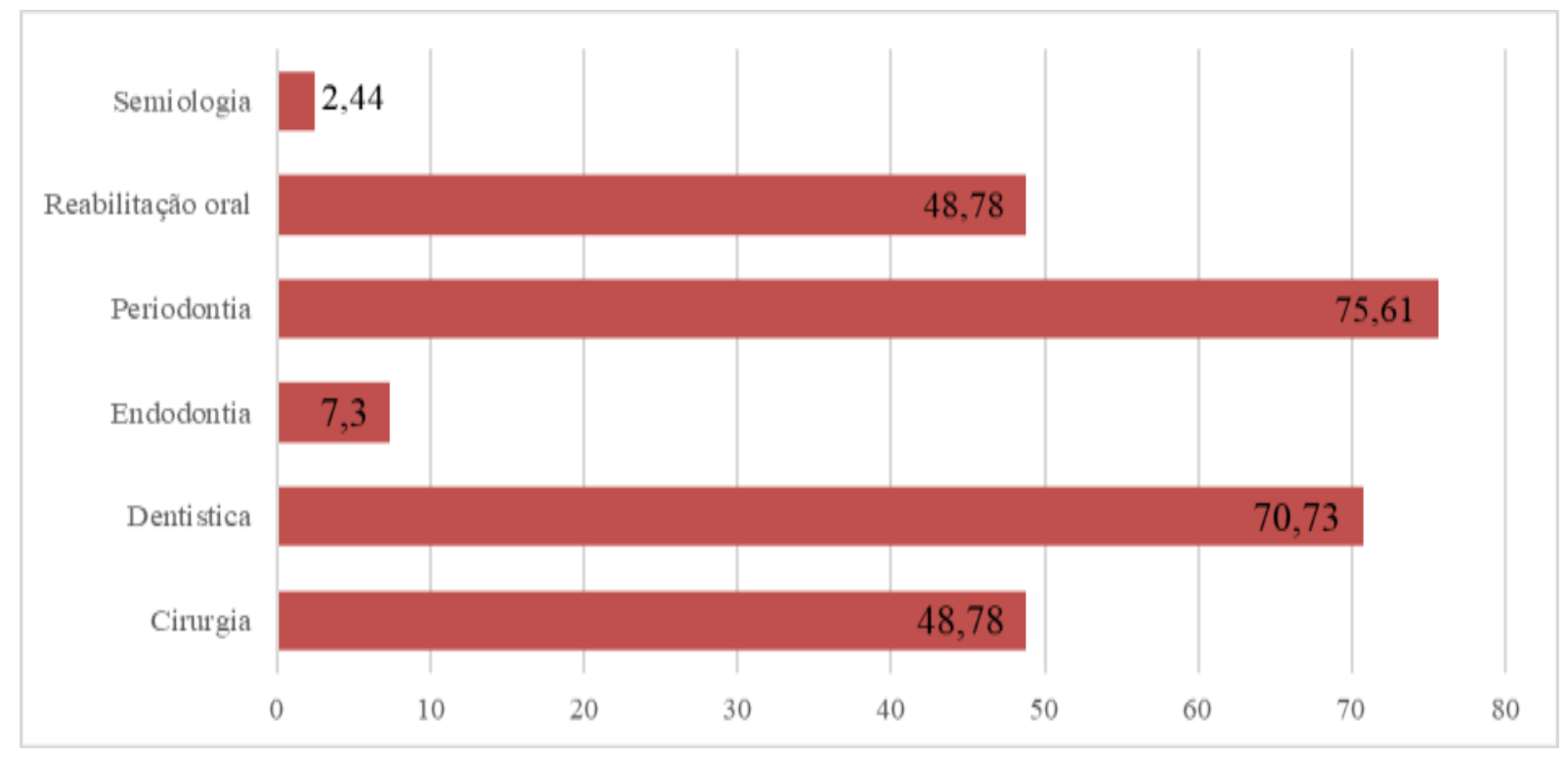

Fonte: Terada et al. (2021).

\section{Discussão}

Este estudo avaliou as condições de saúde bucal de dependentes químicos em processo de recuperação. Dentre as fichas analisadas, 59\% dos indivíduos eram do sexo masculino, corroborando com os dados de Albini et al. (2015), Almeida, Anjos, Vianna e Pequeno (2014) e Falcão et al. (2015), nos quais 86,68\%, 92,2\% e 95,5\% do total de participantes, respectivamente, também eram do sexo masculino.

Quanto à idade, observa-se que o uso e dependência de drogas têm sido cada vez mais precoce. No presente estudo o perfil de idade permaneceu entre 31-40 anos, no entanto, a literatura demonstra-se variável, englobando uma faixa etária que se inicia aos 18, podendo estender-se até mesmo aos 82 anos de idade (Almeida et al., 2014; Falcão et al., 2015). Falcão et al. (2015) relataram que a idade mínima registrada entre os dependentes químicos em estudo foi de 13 anos. Araújo, Costa e Cruz (2018) e Capistrano et al. (2013), observaram que a média de idade do primeiro consumo de drogas ocorreu antes mesmo dos 18 anos de idade.

Em relação a procura por tratamento em clínicas de reabilitação, na presente pesquisa, tal procura aparentou ser maior entre adultos, corroborando com o demonstrado por Almeida et al. (2014) e Falcão et al. (2015). Isso se dá, devido a fase adulta corresponder a uma faixa etária com indivíduos que na maioria das vezes, já tenham constituído família e ingressado no mercado de trabalho, fatores esses, motivadores para a busca por tratamento. De acordo com os dados de Capistrano et al. (2013), dos 350 indivíduos avaliados, 180 (66,2\%) possuíam filhos.

Quanto aos dados das condições de saúde bucal dos dependentes químicos em processo de recuperação, o índice CPO-D encontrado assemelhou-se aos achados de Albini et al. (2015), os quais encontraram CPO-D médio de 15,21. Romão, Falcão, Pereira e Brizon (2016) afirmaram, por meio de revisão de literatura, que o alto índice de CPO-D em indivíduos que fazem o uso de drogas esteve relacionado à negligência dos usuários com cuidados de higiene pessoal, associada às condições vulneráveis as quais a maioria desses pacientes estão expostos, além do consumo de dieta cariogênica, tornando-os mais suscetíveis ao desenvolvimento de cárie. Em estudo para avaliação do perfil nutricional de dependentes químicos em processo de reabilitação, Monteiro, Oliveira, Vilar, Araújo e Carvalho (2020), observaram que dentre os alimentos mais consumidos pelos participantes da pesquisa, encontravam-se os derivados de gorduras e carboidratos, e que o padrão de dieta adotado era pobre em nutrientes, com alta ingestão açúcares. 
Albini et al. (2015) observaram que dependentes químicos com tempo superior a cinco anos de uso de drogas, apresentaram CPO-D significativamente maior $(\mathrm{p}<0,01)$, quando comparados a dependentes que consumiam por menos tempo, demonstrando dessa forma, que além da higiene oral deficiente, composição da dieta, entre outros fatores, o tempo de dependência também pode ser considerado como fator modificador da condição de saúde bucal desses indivíduos.

Ao analisar os resultados do exame periodontal, dados próximos ao observado na presente pesquisa foram relatados por Ferreira et al. (2018), os quais encontraram prevalência de doença periodontal em 51,5\% dos indivíduos examinados e sangramento a sondagem presente em $26,2 \%$ dos pacientes avaliados. No estudo de caso-controle de Arora, Ragi, Arora e Gupta (2019), nenhum dos pacientes do grupo caso (dependentes químicos) apresentaram gengiva saudável, e 56\% deles tiveram sangramento gengival frente a estímulos leves. Dessa forma, é perceptível o quanto condições de vulnerabilidade e ausência de recursos são alguns dos fatores os quais predispõe indivíduos a condições de saúde precária, independentemente do local ou região de estudo.

Sendo assim, no presente estudo, 75,61\% dos indivíduos apresentavam condição de saúde bucal deficiente e cerca de aproximadamente metade dos participantes, 48,78\% $(\mathrm{n}=20)$ declararam ter halitose. No entanto, $63,41 \%$ afirmaram escovar os dentes três vezes ao dia. Costa et al. (2011) notaram que 77,15\% dos participantes avaliados apresentavam saúde bucal deficiente ou precária sendo que, $70 \%$ dos mesmos pacientes, relataram escovar os dentes duas ou três vezes ao dia. Verificaram dessa forma, que a afirmação sobre a frequência de escovação dental não condizia com a situação odontológica observada, assim como na presente pesquisa.

Em relação ao uso do fio dental, $49 \%$ dos pacientes em processo de recuperação afirmaram não fazer uso do fio dental. Ribeiro et al. (2002) observaram que 69,60\% dos examinados não utilizavam o fio dental, e associaram a saúde bucal com a baixa frequência do uso desse instrumento. Shekarchizadeh, Khami, Mohebbi e Virtanen (2013), viram que $81 \%$ dos usuários em tratamento não usavam fio dental e 48\% relataram não escovar os todos os dias. Observa-se dessa forma, que hábitos adquiridos em condições de rua, como se vivem a grande maioria dos dependentes químicos, tornam-se rotina mesmo após o processo de reabilitação nas clínicas de tratamento. Ressalta-se assim a importância de estratégias voltadas ao contexto de saúde bucal a esses indivíduos.

Observou-se também que dentre as condições dos tecidos moles dos participantes avaliados, 39,02\% tinham alterações na mucosa, no entanto estas não foram especificadas. Trabalhos relatam o surgimento de diferentes tipos de lesões, incluindo queimadura nos lábios, úlceras, lesões por mordiscamento, entre outras (Falcão et al., 2015; Colodel et al., 2009). Sendo assim, atenção especial deve ser dada não somente as estruturas dentais e periodontais, sendo essencial inspeção da cavidade bucal em busca de alterações teciduais nesses pacientes.

Por fim, foi visto que boa parte dos dependentes químicos realizou a última consulta odontológica em período superior a cinco anos. Alves et al. (2013) registraram que cerca de $35 \%$ dos pacientes realizaram a última consulta odontológica em um período superior a 3 anos. Na presente pesquisa, em decorrência da quantidade de tempo distante das consultas, a maior parte dos pacientes apresentavam necessidade de tratamentos periodontais, restauradores e de reabilitação oral.

Como limitação, no presente estudo foram feitas apenas análises descritivas, não havendo interação estatística entre as variáveis em estudo, embora, os dados recuperados por meio dos prontuários tenham sido eficazes em demonstrar a real e precária condição de saúde bucal dos dependentes químicos em estudo. Dados como esses são fundamentais como complemento para o desenvolvimento de estratégias educativo-preventivas e de tratamento odontológico voltada a esses pacientes.

Sendo assim, torna-se evidente a relevância de estudos epidemiológicos como ponto estratégico na tomada de decisões para o desenvolvimento de políticas públicas de saúde bucal voltada aos usuários de droga. No entanto, futuras 
pesquisas devem ser realizadas, principalmente àquelas embasadas em dados primários, como forma de aproximar ainda mais seus resultados a real condição de saúde bucal dos indivíduos submissos à dependência química.

\section{Conclusão}

No presente estudo foram observadas condições bucais desfavoráveis e presença de problemas odontológicos entre os indivíduos do estudo, tais como, elevado índice CPO-D, sangramento a sondagem, bolsas periodontais, bem como alterações na mucosa oral. Dessa forma, destaca-se a importância da promoção de saúde bucal em indivíduis em processo de recuperação de dependência química.

\section{Referências}

Albini, M. B., Couto, A. C. F., Invernici, M. M., Martins, M. C., Lima, A. A. S., Gabardo, M. C. L., \& Machado, M. A. N. (2015). Perfil sociodemográfico e condição bucal de usuários de drogas em dois municípios do Estado do Paraná, Brasil. Revista de Odontologia da UNESP, 44(4), $244-249$.

Almeida, R. A., Anjos, U. U., Vianna, R. P. T., \& Pequeno, G. A. (2014). Perfil dos usuários de substâncias psicoativas de João Pessoa. Saúde Debate, $38(102), 526-538$.

Alves, D. M., Nai, G.A., \& Parizi, J. L. S. (2013). Avaliação da ação do uso de drogas na saúde bucal de dependentes químicos. Colloquium Vitae, 5(1), 40-58.

Araújo, R. R. F., Costa, J. V., \& Cruz, V. S., Jr. (2018). A prevalência do uso de bebidas alcoólicas entre adolescentes brasileiros de 13 a 18 anos e sua associação com características demográficas e comportamentais. Research, Society and Development, 7(10), 01-13.

Arora, P. C., Ragi, K. G. S., Arora A., \& Gupta, A. (2019). Oral health behavior and treatment needs among drug addicts and controls in Amritsar District: a case-controlled study. Journal of neurosciences in rural practice, 10(2), 201-206.

Brasil. Secretaria Nacional de Política sobre Drogas (SENAD). (2011). Drogas: cartilha sobre maconha, cocaína e inalantes. Ministério da Justiça. Recuperado em 07 janeiro, 2021, de https://ufsj.edu.br/portal-repositorio/File/lapsam/APA_-_ANPAD.pdf

Capistrano, F. C., Ferreira, A. C. Z., Silva, T. L., Kalinke, L.P., \& Maftum, M. A. (2013). Perfil sociodemográfico e clínico de dependentes químicos em tratamento: análise de prontuários. Escola Anna Nery Revista de Enfermagem, 17(2), 234-241.

Cima, T., \& Côrrea, K. (2014). Alterações bucais em dependentes químicos. Journal of Oral Invest, 3(2), 8-12.

Colodel, E. V., Silva, E. L. F. M., Zielak, J.C., Zaitter, W., Michael-Crosato, E., \& Pizzatto, E. (2009). Alterações bucais presentes em dependentes químicos. Revista Sul-Brasileira de Odontologia, 6(1), 44-8.

Costa, S. K. P., Godoy, G. P., Gomes, D. Q., Pereira, J. V., \& Lins, R. D. A. U. (2011). Fatores sociodemográfcos e condições de saúde bucal em drogadependentes. Pesquisa Brasileira em Odontopediatria e Clínica Integrada, 11(1), 99-104.

Falcão, C. A. M., Santos, R. O., Pereira, R. M. S., Silva, T. S. O., Ferreira, R. S., Silva, S. W. C., Sousa, M. P., \& Ferraz, M. A. A. L. (2015). Saúde bucal em dependentes químicos. Revista Interdisciplinar Ciências e Saúde, 112-121.

Ferreira, W. B., Assis, W. C., Teixeira, W. D., Oliveira, M. F. N., Nunes, L. A., \& Casotti, C. A. (2018). Saúde bucal de usuários de drogas institucionalizados. Revista Enfermería Actual, (35).

Malta, M., Cardoso, L. O., Bastos, F. I., Magnanini, M. M. F., \& da Silva, C. M. (2010). STROBE initiative: guidelines on reporting observational studies. Revista de Saúde Pública, 44(3), 559-565.

Marques, L. A. R. V., Lotif, M. A. L., Rodrigues Neto, E. M., Nunes Neto, A. P., Melo, C. C. S. A., \& Lobo, P. L. D. (2016). Abuso de drogas e suas consequências na saúde bucal: uma revisão de literatura. Revista da Faculdade de Odontologia de Lins, 26(1), 29-35.

Monteiro, N. V. N., Oliveira, I. K. F., Vilar, T. M., Araújo, F. Y. G., \& Carvalho, C. M. R. G. (2020). Perfil nutricional de usuários de entorpecentes em tratamento em um estado da região nordeste. Research, Society and Development, 9(1), 1-9.

Ribeiro, E. D. P., Oliveira, J. A., Zambolin, A. P., Lauris, J. R. P., \& Tomita, N. E. (2002). Abordagem integrada da saúde bucal de droga-dependentes em processo de recuperação. Pesquisa Odontológica Brasileira, 16(3), 239-245.

Romão, D. A., Falcão, A., Pereira, A. C., \& Brizon, V. S. C. (2016). Experiência de cárie dentária em usuário de drogas psicoativas. Revista Brasileira de Pesquisa em Saúde, 18(3), 137-143.

Seemüller, I. V., Klembra, H. L., Toetato, V., Chaiben, C. L., Resende, L. G., Compagnoni, M. M., Machado, M. A. N., \& Lima, A. A. S. (2015). Perfil do paciente em tratamento para dependência química do álcool: uma abordagem odontológica. Revista Extensão em Foco, 11, 39-56.

Shekarchizadeh, H., Khami, M. R., Mohebb, S. Z., \& Virtanen J. I. (2013). Oral health behavior of drug addicts in withdrawal treatment. BMC Oral Health, 13(11), 1-7. 